\title{
Las autoridades de regulación audiovisual en España. Panorámica y perspectivas del control de la pluralidad
}

\author{
MHCJ no 7 | Año 2016 \\ Artículo no 4 (76) \\ Páginas 87 a 112 \\ mhjournal.org
}

\author{
Carlos López Olano | clolano@uv.es \\ Universidad de Valencia
}

\section{Palabras clave}

Autoridad regulatoria; Consejo audiovisual; CNMC; informativos: pluralidad; televisión pública.

Sumario

1. Introducción y objetivos 2 . Metodología

3. Estado de la cuestión 3.1 Una mirada al exterior 3.2 El nonato Consejo Estatal de Medios Audiovisuales. El CNMC asume sus funciones 3.3 Las resoluciones de la CNMC y sus carencias 3.4 Las autoridades de regulación autonómicas $3.5 \mathrm{El}$ marco legal en la Comunitat Valenciana 4. Resultados. Previsiones de futuro 5. Conclusiones 6. Bibliografía 7. Notas

\section{Resumen}

Las autoridades de regulación audiovisual son un instrumento de control, entre otras cosas, de la pluralidad informativa de las televisiones públicas. Así lo reconocen diversas leyes a nivel comunitario y estatal. En España, la asunción de la autoridad en materia audiovisual por parte de la Comisión Nacional de los Mercados y la Competencia (CNMC), nos ha incorporado tarde y mal al entorno europeo, donde son habituales estos consejos. También en las diferentes Comunidades Autónomas, hay un déficit regulatorio que hace que tan sólo Catalunya y Andalucía estén dotadas de este instrumento. En el artículo estudiaremos el "estado del arte" sobre la cuestión, para poder valorar nuestro entorno, con especial atención al caso singular en la Comunitat Valenciana, que se enfrenta a una situación de tabula rasa en materia audiovisual. A pesar de que tanto el Estatut d'Autonomia como la ley audiovisual obligaban a la creación del Consell Audiovisual Valencià desde hace nueve años, el antiguo gobierno de la Generalitat, en consonancia con la política del PP en toda España, bloqueó la iniciativa. Los condicionantes políticos después de las convocatorias electorales de los años 2015 y 2016 influirán sin duda en el panorama futuro respecto a estas herramientas de control.

\section{Forma de citar este artículo en las bibliografías}

Carlos López Olano (2016): “Las autoridades de regulación audiovisual en España. Panorámica y perspectivas del control de la pluralidad”, en Miguel Hernández Communication Journal, nº7, páginas 87 a 112. Universidad Miguel Hernández, UMH (Elche-Alicante). Recuperado el__ de de 20__ de: Џink del artículo en

mhjournal.org] 


\section{Audiovisual regulatory authorities in Spain. Overview and Prospects about the plurality control}

MHCJ no 7 | Año 2016

Artículo no 4 (76)

Páginas 87 a 112

mhjournal.org
Carlos López Olano | clolano@uv.es

Universidad de Valencia
Keywords

Regulatory authority; Audiovisual Council; CNMC; news: plurality; public television.

Summary

1. Introduction and goals 2. Methodology

3. Status of the issue $3.1 \mathrm{~A}$ look abroad

3.2 The unborn Consejo Estatal de Medios responsabilities 3.3 Resolutions of the CNMC and its lacks 3.4 Regional regulatory authorities 3.5 The legal framework in Valencian Community 4. Results. Future foresights 5. Conclusions 6. Bibliography 7.Notes Audiovisuales. The CNMC takes their

\begin{abstract}
Audiovisual regulatory authorities are an instrument of control, among other things, of the plurality of information in public television. This is recognized by so european as national laws. In Spain, the assumption of the authority in the audiovisual field by the National Commission on Competition and Markets (CNMC), has joined us late and wrong to our European environment, where these tips are usual. Also in the spanish regional governments, there is a regulatory deficit: only Catalonia and Andalusia have got this instrument. In the article we will study the "state of the art" on the issue, to value our environment, with special attention to the particular case of Valencia, which is facing a tabula rasa situation in the audiovisual field. Although both the Estatut d'autonomia as regular audiovisual law are requiring the creation of Valencian Audiovisual Council since nine years ago, the former government of the Generalitat, such as the
\end{abstract} policy of the Partido Popular (PP) in Spain, has blocked the initiative. Political conditions after the electoral processes of years 2015 and 2016 could affect in the future state about these control tools.

\section{How to cite this paper in bibliographies}

Carlos López Olano (2016): "Las autoridades de regulación audiovisual en España. Panorámica y perspectivas del control de la pluralidad”, en Miguel Hernández Communication Journal, nº7, páginas 87 a 112. Universidad Miguel Hernández, UMH (Elche-Alicante). Recuperado el de de 20__ de: [link del artículo en 


\section{Introducción y objetivos}

Los organismos independientes como garantes de la pluralidad y el respeto de los valores y la legalidad vigente en el universo audiovisual son habituales en los países de nuestro entorno. Desde finales de la década de los años ochenta, con la 89/552/CEE, ${ }^{1}$ denominada "Directiva sobre Televisión sin Fronteras" (TSF) se aportó una referencia normativa unitaria para los distintos países de la Unión Europea, que realizarían su transposición a lo largo de los noventa. De esa norma, y de las sucesivas directivas que la sustituirían y completarían (Gavaldà Roca, 2011: 78). Pero en España, las transposiciones europeas han sido calificadas como "tardías, laxas, y poco afinadas" (de Moragas \& Prado, 2000: 33).

Uno de los importantes asuntos audiovisuales presentes en la normativa continental es precisamente el de la autoridad regulatoria. La ordenación española de este organismo no llega hasta la Ley General de la Comunicación Audiovisual (LGCA) 7/2010². Pero el retraso en la aplicación de las recomendaciones europeas no acaba ahí. El cambio político después de dos legislaturas de gobierno socialista, al llegar al poder Mariano Rajoy, hace que el nombrado por la ley como Consejo Estatal de Medios Audiovisuales (CEMA) siga sin desarrollarse en inicio, hasta que la Comisión Nacional de los Mercados y la Competencia (CNMC) asume la tarea de regulación audiovisual en el año 2013. Pero en todo el tiempo transcurrido hasta finalizar la revisión de este artículo, en abril de 2016, el denominado macro regulador aún no ha tenido ocasión de evaluar el cumplimiento del servicio público en RTVE.

Las peculiaridades del sistema autonómico español complican aún más la cuestión, ya que el CNMC no tiene competencias en las televisiones de ámbito no estatal. El objetivo del artículo es analizar el estado de la cuestión, tanto en el entorno europeo, como en España. También la valoración de la labor realizada por la CNMC, tres años después de su puesta en marcha, y la situación peculiar en la que se encuentra la Comunitat Valenciana, con un sistema audiovisual inexistente, después del cierre de RTVV ordenado por el gobierno del presidente Alberto Fabra.

\section{Metodología}

La complejidad del asunto que desplegaremos en este artículo hace que creamos conveniente asumir un análisis histórico-organizacional del estado de la cuestión, que nos servirá para valorar mejor las carencias en nuestro panorama audiovisual. La metodología comparativa entre diferentes tipos de organismos similares en países de nuestro entorno, con un estudio cualitativo del marco legal y de las circunstancias sociales y políticas, permitirá también comprender mejor la situación y analizar las previsiones de futuro que existen en esta cuestión. Abordaremos el tema con una metodología descriptiva basada en el estudio de estos reguladores análogos, que permita aplicar su funcionalidad al caso español.

La elección de estos organismos de diferentes países incluidos en el estudio se ha hecho mediante un muestreo no probabilístico de conveniencia, con la intención de ofrecer un marco conjunto adecuado: exceptuando el caso norteamericano, con un modelo totalmente alejado al nuestro, son naciones de nuestro entorno geográfico, cultural y jurídico, con las que además compartimos las 
obligaciones y garantías que emanan del derecho comunitario. También analizaremos descriptivamente la compleja red de leyes nacionales y autonómicas que tienen que ver con la regulación audiovisual, así como su aplicación. Realizaremos finalmente un estudio de caso local, centrado en las peculiaridades del sistema audiovisual de la Comunitat Valenciana, en plena ebullición debido a la situación de tabula rasa provocada por la ausencia de servicio público de radiotelevisión.

\section{Estado de la cuestión}

\subsection{Una mirada al exterior}

Antes de entrar en detalle con las vicisitudes legales por las que hemos pasado en España hasta llegar a la actual situación audiovisual, abrimos el campo para poder tener una referencia de cómo en el exterior se gestionan, desde la perspectiva jurídica, las garantías y los mecanismos de control de la independencia de las televisiones, especialmente de las públicas. Las autoridades que repasaremos serán la estadounidense FCC, el CSA francés, el DLM alemán, el AGCOM italiano y el OFCOM británico. Al caso concreto de la BBC y la completa regulación británica, le dedicamos espacio extra al pensar que es un modelo reconocido mundialmente, de probado éxito, y que puede servir como buen ejemplo.

\section{Federal Communications Commission (FCC) ${ }^{3}$}

El organismo estadounidense se creó en 1934. Empezó con competencias de radio, después de telecomunicaciones y más tarde de televisión. Mira especialmente por el pluralismo externo, es decir que el mercado de la comunicación sea libre, para lo que pueden establecerse limitaciones a la propiedad: normas anticoncentración más estrictas que las que protegen la libre competencia. Pero este entramado de protecciones se ha ido desmontando desde la época del presidente Ronald Reagan en aras de la rentabilidad de las empresas (Lakidain \& Patterson, 2012: 131).

Hay que tener en cuenta el muy diferente mercado audiovisual norteamericano respecto al europeo: aunque sí que existe la televisión pública -Public Broadcasting Service (PBS)- que se fundó en 1970 y disfruta de una considerable calidad y prestigio, la cadena tiene generalmente una audiencia poco más que testimonial, por lo que las grandes cadenas privadas no ven amenazado su terreno. El FCC mantiene un alto grado de independencia con relación al Gobierno y las cámaras legislativas, aunque no se ha librado de las críticas en los últimos años, por parcialidad con las empresas del sector. Tiene cinco comisarios -antes eran siete, pero se redujeron por recortes en el presupuesto- nombrados por el presidente de los EUA con la aprobación del Senado para siete años de mandato. A la FCC le corresponde como función esencial, otorgar las licencias de emisión de radios y televisiones, previa constatación de un conjunto de requisitos jurídicos, técnicos y financieros de obligado cumplimiento por las empresas solicitantes de una determinada frecuencia.

En los supuestos de que una frecuencia se haya solicitado por más de un peticionario, una comisión examinará cuál de las opciones es la más cualificada y responde mejor a los intereses del 
público para decidir a quien se la otorga. Dichas licencias que inicialmente se concedían por un periodo de tres años, se ampliaron mediante una Ley de 1981 a cinco años para la televisión y siete para las de radio. Los solicitantes de estas licencias pueden ser personas físicas o jurídicas, no existiendo disposición alguna restrictiva al respecto, salvo la prohibición de otorgarlas a sociedades de capital extranjero o a sociedades que tengan más de un $20 \%$ de acciones propiedad de extranjeros.

\section{Conseil Supérieur de l'Audiovisuel (CSA) ${ }^{4}$}

Creado en Francia en 1989 para garantizar la libertad en la radiodifusión. Es la autoridad audiovisual europea con más competencias y poderes. Está formada por siete ${ }^{5}$ miembros de los cuales el presidente es nombrado por el presidente de la República. El presidente de la Asamblea Nacional y el del Senado nombran cada uno tres miembros, pero con un previo dictamen de conformidad de al menos tres quintas partes de los miembros de las respectivas comisiones encargadas de asuntos culturales. Entre sus múltiples atribuciones, está el velar por la calidad de los programas, por la defensa y promoción de la lengua y la cultura, controlar la publicidad, las campañas electorales, intervenir en la gestión y atribución de frecuencias para radio y televisión, y dictar medidas coercitivas contra las infracciones.

Algunos de los objetivos del CSA son garantizar la libertad de comunicación así como la igualdad de trato, la independencia y la imparcialidad del sector público de radiodifusión y televisión; promover la libre competencia; velar por la calidad y diversidad de la emisión, el desarrollo de la producción nacional y la defensa de la lengua y la cultura francesa (art. 1); ordenar, también en el sector público, las emisiones durante las campañas electorales (art. 16); la gestión del espacio radioeléctrico y la autorización para la utilización de frecuencias para la emisión de radio y televisión por ondas hertzianas, así como para la televisión por satélite (art. 22). En resumen podemos decir que al Consejo Superior del Audiovisual francés le corresponde la regulación, tutela y control del sector, tanto público como privado.

También destaca entre sus amplias e importantes funciones, el nombramiento por mandatos de tres años de cuatro miembros, entre ellos el presidente, de los consejos de administración de las radios y de las cadenas públicas de televisión (Tornos Mas \& de Carreras i Serra, 1999: 101).

La oposición ha acusado de parcialidad recientemente al actual presidente Françoise Hollande, ya que que a pesar de todos los cambios normativos, el presidente del CSA es un reconocido socialista y antiguo colaborador de Lionel Jospin. ${ }^{6}$

\section{Direktorenkonferenz der Landesmedienanstalten (DLM) ${ }^{7}$}

Autoridades reguladoras de la difusión de Alemania. Cada Land -estado federado- tiene una autoridad, en línea con la tradición descentralizadora germana, que regula la radiodifusión privada y pública. Todos estos 14 organismos se agrupan en DLM. Entre sus competencias están la concesión y la asignación de licencias, y el control de contenidos y de la publicidad. Las 
competencias sobre cultura y radiotelevisión corresponde a los Länder, y las telecomunicaciones en general al gobierno federal. Las emisoras públicas -equivalentes a las autonómicas españolasestán reguladas por los respectivos consejos de radiodifusión.

\section{Autorità per le garanzie nelle comunicacioni $(\mathrm{AGCOM})^{8}$}

Organismo italiano regulador creado en 1997. Formado por un presidente, la comisión para infraestructuras con cuatro miembros, la de los servicios y los productos con otros cuatro miembros, y el consejo formado por el presidente y todos los consejeros. Estos son elegidos por el Senado y la Cámara de Diputados. Entre sus competencias, está el participar en la elaboración del Plan Nacional de distribución de frecuencias, registrar los operadores de comunicación, determinar el ámbito de las eventuales obligaciones de servicio universal, o intervenir en las controversias entre el ente gestor del servicio de telecomunicaciones y los usuarios privados. También tiene que controlar la publicidad, la protección de los menores y las minorías lingüísticas, y garantizar la aplicación de normas sobre propaganda política y electoral.

La RAI tiene su propio sistema de regulación, al margen del AGCOM, el Consejo de Administración es el máximo órgano de gestión y el responsable del seguimiento de las obligaciones de servicio público por la corporación.

\section{Office of Communications (OFCOM) ${ }^{9}$}

Macroentidad reguladora del Reino Unido, que recogió las funciones de varios organismos anteriores. Fue ordenada por una Communications Act en el año 2003, y recogió las competencias por ejemplo de la antigua Independent Television Commission (ITC), de la Broadcasting Standards Commission (BSC), y de la Office of Telecommunications (OFTEL). Las competencias en telecomunicaciones están en sus manos, y regula la industria de la radio y la televisión, las líneas fijas de teléfono, los móviles, y los servicios postales.

El OFCOM supervisa algunas parcelas de la BBC, y esta tiene que cumplir el código de esta institución externa, aunque las cuestiones relativas a la imparcialidad o la exactitud, o sobre elecciones o referéndums, quedan bajo la custodia del BBC Trust. Las tensiones han sido inevitables, en una situación en la que los periodistas están sujetos a dos códigos distintos según su trabajo se desarrolle en la sede de la BBC $^{10}$ o fuera de ella (Belando Garín, 2014: 131). la confusión llega al espectador, que en caso de querer formular una queja, debe hacerlo al OFCOM en caso de ser relativa a la protección de la infancia, y al BBC Trust en caso de tener que ver con la imparcialidad. Este último organismo es el garante de que "the BBC remains independent, resisting pressure and influence from any source” (BBC, 2006, 19.2.1).

Más allá de estas dificultades organizativas del cruce de distintos organismos de control, dentro del ámbito de los contenidos el punto central de referencia ha sido la delimitación de lo que debía considerarse como "servicio público" y la necesidad de convertirlo en el eje de las programaciones del conjunto de emisiones de la $\mathrm{BBC}$. Al respecto debe precisarse que este 
concepto, construido sobre la base práctica de los primeros años de funcionamiento de una empresa casi centenaria, ha ido desarrollándose y matizándose conforme ha ido transformándose la sociedad y la audiencia; al tiempo que su aplicación práctica ha sido el resultado de una interacción entre políticos, periodistas y gestores de los medios.

Y el Reino Unido, no lo olvidemos, ha sido considerado "the historic heartland of Public Service Broadcasting” (Lowe \& Steemers, 2012: 9). Desde los postulados de John Reith, mítico primer director de la cadena, que asociaba el servicio público a un celo moral sobre las emisiones un tanto paternalista, procurando incluir lo que se considerara bueno para la sociedad y dejando fuera lo malo con la calidad como primera norma, hasta concepciones más contemporáneas como las que se incluyen en el "Informe del Comité Annan" de 1977 en el que se rechaza que los gestores de la televisión puedan imponer un modelo de objetivos morales y sociales; o los planteamientos del "Comité Peacock" de 1985, en el que se acerca la idea de servicio público a los intereses del conjunto de la audiencia y de los ciudadanos como contribuyentes (Linde Paniagua \& Vidal Beltrán, 2003).

En la era de la convergencia digital y la hibridación de medios a través de los cuales llegan los contenidos audiovisuales, desde el seno de OFCOM se ha reclamado que su regulación alcance también la difusión a través de internet y redes, proponiendo la autoregulación como única alternativa dentro de un contexto de rápida evolución del mercado (Ariño, 2008).

\begin{tabular}{|c|c|c|c|c|c|}
\hline $\begin{array}{l}\text { Nombre } \\
\text { organismo }\end{array}$ & FCC & CSA & DLM & AGCOM & OFCOM \\
\hline País & $\begin{array}{l}\text { Estados } \\
\text { Unidos }\end{array}$ & Francia & Alemania & Italia & Reino Unido \\
\hline $\begin{array}{ll}\text { Fecha de } \\
\text { fundación }\end{array}$ & 1934 & 1989 & 1987 & 1997 & 2003 \\
\hline Atribuciones & $\begin{array}{l}\text { Vela sobre } \\
\text { todo por } \\
\text { pluralismo } \\
\text { externo y } \\
\text { protección } \\
\text { competencia, } \\
\text { concede y } \\
\text { renueva las } \\
\text { licencias }\end{array}$ & $\begin{array}{l}\text { Muy } \\
\text { amplias, } \\
\text { regula la } \\
\text { concesión de } \\
\text { licencias e } \\
\text { incluso } \\
\text { nombra al } \\
\text { presidente } \\
\text { de las } \\
\text { cadenas } \\
\text { públicas }\end{array}$ & $\begin{array}{l}\text { Estructura } \\
\text { atomizada. } \\
\text { Coordina } 14 \\
\text { organismos } \\
\text { de control } \\
\text { autónomos, } \\
\text { uno por cada } \\
\text { Land. } \\
\text { Conceden y } \\
\text { controlan las } \\
\text { licencias }\end{array}$ & $\begin{array}{l}\text { Participa en } \\
\text { distribución } \\
\text { de licencias. } \\
\text { Comparte } \\
\text { atribuciones } \\
\text { de control } \\
\text { con } \\
\text { organismo } \\
\text { interno RAI }\end{array}$ & $\begin{array}{l}\text { Regula la } \\
\text { TV, } \\
\text { teléfono y } \\
\text { los servicios } \\
\text { postales. } \\
\text { Comparte } \\
\text { control con } \\
\text { BBC Trust }\end{array}$ \\
\hline $\begin{array}{l}\text { Garantías de } \\
\text { independencia }\end{array}$ & $\begin{array}{l}\text { Consejeros } \\
\text { nombrados } \\
\text { por }\end{array}$ & $\begin{array}{l}\text { Consejeros } \\
\text { nombrados } \\
\text { por }\end{array}$ & $\begin{array}{l}\text { Consejeros } \\
\text { nombrados } \\
\text { por }\end{array}$ & $\begin{array}{l}\text { Consejeros } \\
\text { elegidos por } \\
\text { el Senado y }\end{array}$ & $\begin{array}{l}\text { Presidente y } \\
\text { consejeros } \\
\text { no }\end{array}$ \\
\hline
\end{tabular}




\begin{tabular}{|l|l|l|l|l|l|}
\hline & $\begin{array}{l}\text { presidente } \\
\text { EUA, con } \\
\text { aprobación } \\
\text { del Senado }\end{array}$ & $\begin{array}{l}\text { Asamblea yidencia, yenado } \\
\text { Senádentes }\end{array}$ & la Cámara & $\begin{array}{l}\text { ejecutivos } \\
\text { nombrados } \\
\text { por el el } \\
\text { ministro de } \\
\text { Cultura }\end{array}$ \\
\hline
\end{tabular}

Tabla 1: resumen organismos de regulación audiovisual

\subsection{El nonato Consejo Estatal de Medios Audiovisuales. La CNMC asume sus funciones}

España ha sido el país de la CE que más ha tardado en crear un ente regulador que tal como acabamos de ver, es común en las democracias desarrolladas. En este epígrafe comprobaremos además las singulares circunstancias en que se ha realizado finalmente esa creación. Las autoridades de regulación cumplen usualmente dos funciones básicas, la regulación de los mercados, y la de los contenidos (Tornos Mas \& de Carreras i Serra, 1999: 19). Las estructuras tradicionales para garantizar el pluralismo en medios públicos han sido los Consejos de Administración y las Comisiones de control del Congreso o de las Cortes autonómicas. Pero frente a estos, amparada por las directivas Europeas, surge la obligación de los Consejos Audiovisuales.

Si nos centramos en las AAI (Autoridades Administrativas Independientes) en el sector del audiovisual, podemos afirmar que su creación responde a la necesidad de separar al regulador del regulado, a la necesidad de actuar neutralmente en la ordenación del sector y a la ineficacia de la administración tradicional para ejercer la nueva función de regulación del sector. (Tornos Mas \& de Carreras i Serra, 1999: 80)

La autorregulación es una opción a considerar, aunque "como consecuencia de un consenso interno y libre está supeditada a las personas [...] por lo que en medios de comunicación donde las directivas están politizadas o condicionadas al mercado, puede que no se dé el ambiente propicio (López Rico, 2013). La creación del Consejo llegó precedida de polémica. Autores como Cabral y Roldán (2011) defendieron una autoridad convergente, siguiendo el modelo británico e italiano, (Ofcom y Agcom). También García Castillejo (2010) abogaba por unificar la Comisión del Mercado de Telecomunicaciones (CMT) previa y el futuro Consejo Estatal de Medios Audiovisuales (CEMA), ordenado como hemos visto en la Ley 7/2010 (LGCA) de 31 de marzo. Finalmente, es mediante la Ley de Creación, la 3/2013, de 4 de junio, ${ }^{11}$ como se pone en marcha la CNMC, ${ }^{12}$ aglutinando varios entes reguladores, entre ellos el comentado CEMA.

El funcionamiento efectivo se inició el 7 de octubre de 2013, y de esta manera se produce la transposición de las directivas comunitarias, en especial la última, 2010/13/UE de 10 de marzo. Aparte del retraso en la llegada del regulador, se ha criticado que este lo haya hecho de una forma descafeinada, entre otros motivos por la concentración de tantas áreas bajo un mismo ente. El 
Partido Popular explicó la medida por la necesidad de ahorro ocasionada por la crisis, y basándose en los modelos de autoridades como la británica OFCOM que acabamos de ver, que también regulan otros sectores.

Esa non nata autoridad independiente se subsume en la Comisión Nacional de los Mercados y la Competencia, junto con las competencias de las suprimidas autoridades de regulación de las telecomunicaciones, la energía, el transporte, el correo postal y la propia autoridad anterior de la competencia, aunque tal supresión chocara frontalmente con la doctrina europea de autoridades independientes en campos muy diferentes y recibiera críticas serias de instancias europeas. Además, su proclamación como 'independiente', es altamente cuestionable porque sus diez miembros son nombrados por el gobierno, a propuesta del Ministerio de Economía, y ratificados por el Congreso por mayoría simple. (Bustamante, 2014: 25-26)

Este procedimiento choca radicalmente con el que estaba previsto en la LGCA, en el que la propuesta de nombramientos provenía del Congreso de los Diputados, y donde era necesaria una mayoría de tres quintas partes de votos para su aprobación. Como afirma con supuesta inocencia el aún vocal asesor de la Secretaría de Estado de Comunicaciones José Luis García, "Finalmente, es posible que no se optase por este tipo de mecanismos o mayorías para no bloquear desde el inicio la constitución de la CNMC" (García López, 2014: 158). Por el camino, se perdieron los mecanismos que aseguran la independencia de los medios y los reguladores, y ese consenso que debe ser considerado como un garante de la democracia y no un mero obstáculo molesto para la tramitación de las leyes.

Además, el Gobierno se convierte en el guardián efectivo de la radiotelevisión pública y de la privada, incluso directamente, ya que competencias como el catálogo de acontecimientos de interés general vuelven a depender de Presidencia del Gobierno, mientras las licencias privadas y las sanciones contra ellas se desplazan al Ministerio de Industria. Las funciones de la CNMC en el campo audiovisual se limitan a un solo artículo, el 9 de la Ley 3/2013, centrado en el control de las cuotas de obras europeas y de financiación anticipada, a los derechos del menor y los códigos de autorregulación, aunque eso sí, incluyen "la vigilancia del cumplimiento de la misión de servicio público”. En la Comisión se establecen cuatro grandes Direcciones de Instrucción:

1. de Competencia

2. de Energía

3. de Transportes y del Sector Postal

4. de Telecomunicaciones y del Sector Audiovisual

A esta última es a la que corresponde, a través de la Subdirección del Audiovisual, las tareas de supervisión y control de los mercados de comunicación audiovisual. Vemos que las funciones 
que había de cumplir el CEMA, quedan desde luego diluidas en una gran cantidad de campos en los que ahora tiene que ejercer la regulación la CNMC. Las competencias de control, además, han de ceñirse a los servicios audiovisuales con cobertura estatal, o al menos que afectan a más de una comunidad autónoma (García López, 2014: 157).

Para Josep Gavaldà, la sustitución del CEMA por esta CNMC con atribuciones tan variadas también hace que España de nuevo se aleje de Europa, ya que:

Los estados de la UE que han constituido organismos reguladores similares a la CNMC, Alemania y Holanda, por ejemplo, han excluido de los mismos la supervisión del audiovisual, porque, como ha insistido hasta la saciedad la literatura regulatoria europea, primero en relación con la radiodifusión y luego con los servicios audiovisuales, su regulación como mercado no puede soslayar en ningún caso su "valor cultural". (2014: 115)

Ya hemos visto que una de los cosas que la CNMC tiene que vigilar es el cumplimiento de la misión de servicio público encomendada a los prestadores del servicio de comunicación audiovisual de ámbito estatal, por ejemplo RTVE. La función estaba ya prevista en la Ley 17/200613, en el Mandato-marco de la Corporación publicado en el BOE no 157 de 30 de junio de 2008, ${ }^{14}$ y también recogida en la LGCA. Hablaremos de ello más ampliamente en seguida. Es una función eso sí, compartida con las Cortes Generales, que ejercen el control parlamentario que impone la Carta Magna.

Otra función destacada es la relativa a los derechos de los menores. Los contenidos que puedan resultar perjudiciales para el desarrollo físico, mental o moral de los menores solo podrán emitirse en abierto entre las 22 y las 6 horas, debiendo ir siempre precedidos por un aviso acústico y visual. El indicador visual deberá mantenerse a lo largo de todo el programa en el que se incluyan dichos contenidos. Asimismo, se establecen tres franjas horarias de protección reforzada: entre las 8 y las 9 y entre las 17 y las 20 horas los días laborables, y entre las 9 y las 12 los sábados, domingos y fiestas de ámbito estatal. La Ley otorga gran importancia además:

al impulso de los códigos de conducta y al establecimiento de una correcta calificación por edades de los programas que se emitan, en función de los criterios homologados por el Código de Autorregulación sobre Contenidos Televisivos e Infancia. Sin embargo, corresponde a la CNMC la vigilancia, control y sanción de la adecuada calificación de los programas efectuada por los prestadores del servicio de comunicación audiovisual televisiva. (García López, 2014:162-163)

La CNMC debería cumplir al menos estas funciones, según Martínez Otero:

1. Establecer formas de inspección y control regulares y eficaces. 
2. Habilitar un sencillo canal de quejas de los ciudadanos.

3. Controlar la adecuación de la calificación de los contenidos, es lo que Martínez considera que es el "núcleo del sistema de protección de los menores" y que "garantizar que esta clasificación sea adecuada se convierte en una prioridad absoluta. Un sistema de restricción de emisiones que operase a la perfección en un contexto en que las clasificaciones de contenidos fueran fruto del arbitrio, capricho o conveniencia de los emisores, no pasaría de ser una burla al derecho y a los televidentes" (2014: 262).

Estas obligaciones serán efectivamente, las bases de las recientes actuaciones regulatorias de la CNMC que enseguida repasaremos.

\subsection{Las resoluciones de la CNMC y sus carencias}

Lo cierto es que después de unos inicios nada alentadores, sumida en críticas generalizadas por su falta de atribuciones y competencias, la CNMC está obligada a mejorar su Auctoritas, que al iniciar su andadura era casi nula. Desde la creación aún reciente del organismo o mejor dicho, de la asunción por éste de las competencias audiovisuales a partir de junio de 2013, fecha de la aprobación de la ley 3/2013, se han producido unas cuantas resoluciones e informes de los que vamos a dar cuenta aquí. El 4 de octubre se completa el organigrama de la CNMC, con el nombramiento de la directora de Telecomunicaciones y del Sector Audiovisual, Alejandra de Iturriaga Gandini, y el 7 de octubre se dicta la Orden Ministerial que la pone en marcha.

El primer estudio relativo al sector, es el correspondiente al segundo trimestre que se publica el 27 de octubre de 2013. Observando la frecuencia de los expedientes, se puede observar como poco a poco la maquinaria reguladora va cogiendo velocidad. Manteniendo el orden cronológico, la siguiente actuación ya es de más calado: se trata de la multa por infracción muy grave con 15 millones de euros a Mediapro y 4 clubes de fútbol ${ }^{15}$ que se impone el 2 de diciembre. Se trata en realidad de la resolución de un expediente anterior incoado por el Consejo de la Comisión Nacional de la Competencia (CNC), por no cumplir las normas en los contratos de retransmisiones de partidos de fútbol. Hay que esperar hasta el 25 de marzo ya de 2014, para ver el primer caso propio del nuevo CNMC: son dos expedientes sancionadores a Mediaset y Atresmedia por emitir contenido inadecuado para menores. ${ }^{16}$

Hay que destacar que en el listado de actuaciones de la CNMC las relativas al audiovisual aparecen diseminadas entre una extensa enumeración de expedientes relativos al precio de la bombona de butano, las liquidaciones del sector eléctrico, el precio de la leche, los ahuyentadores de plagas o de la gestión de los derechos intelectuales: son los inconvenientes inherentes a los macrorreguladores. El 4 de septiembre la CNMC impone sanciones a Mediaset, Atresmedia y Net TV por valor de 386.902 euros por incumplir diversos artículos de la LGCA, que marcan los tiempos máximos de publicidad y el tipo de contenidos que se pueden emitir en determinados horarios, y el 12 del mismo mes, otra a Mediaset de 111.000 euros por confundir publicidad y contenidos. ${ }^{17}$ El 24 de octubre, sanciona a Atresmedia por emitir contenido inadecuado para menores, en concreto por el programa "Física o Química" (calificado de +7). ${ }^{18}$ 
Dentro de este resumen de actuaciones, destaca por sus implicaciones y su impacto mediático la "Resolución por la que se requiere a Mediaset España Comunicación, S. A. para que adecúe la calificación y emisión del programa 'Sálvame diario' a lo establecido en el código de autorregulación sobre contenidos televisivos e infancia, y a la Ley 7/2010" que se publicó el 17 de diciembre de 2014. ${ }^{19}$ La resolución responde a una denuncia de la Asociación de Usuarios de la Comunicación, debido a que dicho programa, un infoshow dedicado principalmente a temas de corazón con un considerable éxito de audiencia, y paradigma de la telebasura en España, ocupa la franja de protección reforzada de la infancia establecida en el Código de Autorregulación firmado por todas las cadenas del país. Concretamente, se incumplirían entre otros, estos criterios establecidos en el anexo al Código:

- No presentación de lenguaje soez expresado de manera ofensiva y/o violenta.

- Evitar la incitación a los niños a la imitación de comportamientos perjudiciales o peligrosos para la salud, especialmente: la incitación al consumo de cualquier tipo de droga y el culto a la extrema delgadez.

- Evitar la utilización instrumental de los conflictos personales y familiares como espectáculo, creando desconcierto en los menores.

Dicho informe de la CNMC, que incluye una amenaza de sanción si en diez días no se ponía remedio a las infracciones, hace referencia a situaciones producidas durante ese horario infantil protegido como la siguiente:

Belén Esteban en tono amenazante: "Que le digas a tu suegra y a tu mujer que me dejen en paz ya, y si me tienen que poner una demanda que me la pongan ya. Que me la pongan ya, pero que a mi madre, cuidadito, porque tiro de la manta, y tiro de la manta. A mi madre ni tocarla, ni a mi hija, porque por mi madre y por mi hija jmato!" [...] “A mí ya todo me da igual, pero ésta no se ríe de mí y menos de mi madre. Porque ahora lo vuelvo a repetir aunque lo saque Ana Rosa: te saco los ojos, me los como, los vomito y los pisoteo, ¿'vale?"

$\mathrm{El}$ informe consiguió un gran revuelo mediático, especialmente por la actitud del presentador y director del programa "Sálvame diario", Jorge Javier Vazquez, que dijo, aprovechando la palestra de su programa de gran audiencia, que todo respondía a una campaña de acoso orquestada por "organizaciones ultraconservadoras" y que "Si un día nos vamos que sea porque ya no nos veis, no porque se decida en un despacho desde fuera de la televisión". El discurso victimista llegó al extremo de calificar el informe de "caza de brujas". ${ }^{20}$

La batería mediática de repulsa al toque de atención de la CNMC llegó también a las redes sociales, con el hashtag \#MásSálvamequenunca en apoyo del programa. La encendida defensa de Vázquez en la que alude aunque sea veladamente al derecho a la información, dejó afirmaciones como ésta: 
No es agradable recibir amenazas como la de hoy pero sí me gustaría dar las gracias a los que quieren echarnos de la tele porque nos han dado la oportunidad de ser conscientes de lo unida que está esta gran familia ante la adversidad.

La postura de la CNMC no se arredró en absoluto ante las críticas, ya que además la airada defensa que hizo Vázquez y sus colaboradores provocaron en las redes sociales más hilaridad, que una percepción de que la democracia estaba amenazada. La "gran familia" en cualquier caso, más allá de los aspavientos iniciales, reaccionó haciendo marcha atrás de forma rápida y efectiva. La solución de Mediaset para intentar cumplir con las denuncias del informe y evitar la amenaza de cierre -tan sólo cinco días después del ultimátum- fue partir el programa en dos, con dos nombres similares pero distintos: "Sálvame limón", para el horario de 16:00 a 17:00 -para mayores de 13 años- sin restricciones para la temática soez, y "Sálvame naranja”, de 17:00 a 20:00, - para menores de esa edad- con contenidos suavizados. Con esto, y con la promesa de respetar el código de autorregulación a partir de entonces, quedó zanjada la cosa de momento, aunque las nuevas denuncias y multas consecuentes no se han hecho esperar. El 6 de abril de 2016 la CNMC sancionó a Mediaset con cerca de 200 mil euros por no respetar de nuevo el horario de protección de menores en el mismo programa. Más sanciones, no son, evidentemente, descartables.

Además de este caso emblemático, en los últimos meses desde el organismo regulador se ha producido una avalancha de informes y denuncias contra las televisiones privadas, eso sí, siempre centradas en el no cumplimiento de estos códigos de infancia y de publicidad. El mismo programa "Sálvame diario" estuvo en el punto de mira de nuevo en un expediente sancionador al día siguiente del primer informe, por emitir un microespacio de salud al que acusaron de publicidad encubierta. El 13 de enero de ya 2015, se conoció otra multa a Mediaset de 112.100 euros por emitir la película "American Playboy” en horario de protección a menores. El 25 de febrero, nuevo expediente sancionador a Mediaset por incumplimiento de la calificación por edades en el programa "Crónicas carnívoras", el 5 de marzo otra vez por publicidad encubierta en el programa de María Teresa Campos "Qué tiempo tan feliz".

Llama la atención que toda esta hiperactividad de la CNMC, y las sanciones a las que el sector no está acostumbrado, están basadas tan sólo en los dos códigos de autoregulación ya comentados, el relativo a la infancia y el de la publicidad. Bienvenidas sean las actuaciones en este campo y el fin de la impunidad, pero se echa en falta otra de las funciones que tiene encomendada la autoridad: tiene que vigilar como hemos visto el cumplimiento de la misión de servicio público de RTVE, tal como manda la Ley 17/2006, el Mandato-marco de junio de 2008, y la Ley 7/2010 General de la Comunicación Audiovisual. En ese tema de momento, parece que no han apreciado ninguna irregularidad. Y eso que el presidente de la CNMC, José María Marín Quemada, anunció en abril de 2015 que el primer informe sobre el cumplimiento de las funciones de servicio público por parte de TVE llegaría en el tercer trimestre del año. Lo hizo en el Fòrum de la Comunicació, organizado por el Consell de l'Audiovisual de Catalunya. ${ }^{21}$ 
La fecha era pertinente: antes de las elecciones generales del 20 de noviembre de 2015. Desgraciadamente, sin que el macroregulador haya dado ninguna explicación, el informe no se publicó a tiempo. Y en el momento de finalizar este artículo, ya bien entrado 2016, sigue sin haber noticias de él.

\subsection{Las autoridades de regulación autonómicas}

Ya hemos visto que la CNMC sólo tiene competencias en televisiones cuya emisión abarque al menos dos comunidades distintas españolas, lo que en teoría deja fuera de su campo de regulación todas las televisiones autonómicas, tanto públicas como privadas, así como las locales. ${ }^{22} \mathrm{El}$ sistema autonómico tendría que cubrir esta laguna con leyes y organismos propios, pero ahora mismo esto sólo ocurre en Cataluña y Andalucía, únicas comunidades en las que la Autoridad Audiovisual está en marcha. Autores como García Castillejo recomendaban incluso la creación de una comisión de cooperación audiovisual para coordinar estos organismos autónomos (2010). En otras comunidades, también en la valenciana, se recogió la idea de su creación -incluso en los Estatutos de Autonomía- pero a pesar de ello los distintos gobiernos autonómicos del PP han desactivado por todos los medios políticos posibles estas iniciativas que estaban en diferentes puntos de ejecución. Incluso la institución reguladora más veterana del país, el CAC, ha pasado por diversas etapas con cambios de calado en las atribuciones que disfruta. Veámoslo con más detalle.

\section{Consell de l'Audiovisual de Catalunya (CAC) $)^{23}$}

Fue creado en 1996 como órgano asesor del gobierno de la Generalitat, pero la reforma legislativa con la 2/2000 de 4 de mayo $^{24}$ es la que lo transformó en una auténtica autoridad regulatoria, con facultades reglamentarias y sancionadoras tanto para la competencia entre operadores de radio o televisión -especialmente en el tema de concesión y reparto de licenciascomo respecto a los contenidos de la programación: publicidad, protección de los menores ante programaciones eventualmente lesivas, discriminación y pluralismo político. Está ordenada además en la Ley 22/2005, de 29 de diciembre, de la Comunicación Audiovisual de Catalunya ${ }^{25}$ que ha sido modificada varias ocasiones. Sus miembros son designados por el Parlament de Catalunya por una mayoría de dos tercios de los votos y debían tener, cada uno de ellos, el apoyo de al menos dos grupos parlamentarios. Tienen un mandato de seis años, sin poder ser reelegidos ni cesados, y su actividad profesional es incompatible con cualquier otra o con intereses en el sector.

El CAC es independiente del Gobierno y del Parlamento, y tiene potestad para regular las emisiones de carácter autonómico -nunca las que tienen un alcance nacional- aunque también tengan emisión en el territorio de Catalunya. Pero no todo son buenas noticias, al repasar la historia de esta institución. La reforma de la CAC realizada mediante la Ley de la Generalitat Catalana 2/2012 de Modificación de varias leyes en Materia Audiovisual de 22 de febrero, ${ }^{26}$ fue calificada también como "contrarreforma" por algunos medios y sindicatos. ${ }^{27}$ Redujo de diez a seis los miembros, y establece que el presidente sea escogido por la Cámara catalana. Consultando la literalidad del cambio legal, así es como estaba el artículo 4 en la Ley 2/2000: 
1. El Consell de l'Audiovisual de Catalunya és integrat per deu membres, nou dels quals són elegits pel Parlament, a proposta, com a mínim, de tres grups parlamentaris, per una majoria de dos terços. L'altre membre, que n'és el president o presidenta, és proposat i nomenat pel Govern després d'escoltar l'opinió majoritària dels nou membres elegits pel Parlament.

2. Els membres del Consell són escollits d'entre persones de prestigi reconegut i contrastat i amb experiència professional en el sector audiovisual, i que ofereixen garanties plenes d'independència.

Y en la modificación de la comentada 2/2012, quedó de esta manera:

4. El Consell de l'audiovisual de Catalunya és integrat per sis membres: cinc consellers i un president o presidenta.

5. Els membres del Consell són escollits d'entre persones de prestigi reconegut i contrastat i amb experiencia profesional en el sector audiovisual, i que ofereixen garanties plenes d'independència.

6. Els membres del Consell són elegits pel Parlament a proposta, com a mínim, de dos grups parlamentaris, per una majoria de dos terços. Si en una primera votació no s'obté la majoria de dos terços, són elegits per majoria absoluta en una segona votació, que s'ha de fer en la mateixa sessió.

7. El president o presidenta del Consell és escollit pel Parlament, per una majoria de dos terços, d'entre els sis membres que integren el Consell. Si en una primera votació no s'obté aquesta majoria de dos terços, és elegit per majoria absoluta en una segona votació, que s'ha de fer en la mateixa sessió.

Vemos que son cambios significativos, similares a otros que el PP incorporó en la legislación estatal: primero reducción del número de miembros, con la excusa de la crisis, es un movimiento generalizado en todo tipo de organismos. Por otra parte, introduce la prevención de que si no hay acuerdo político en un primer momento, en una segunda votación sólo hará falta la mayoría absoluta. Esta modificación en la norma provoca la desaparición, claro está, de la necesidad de consenso. Al menos, en la reforma de la Ley 17/2006 para nombrar al presidente de la Corporación de RTVE que proponía maniobra similar para evitar los bloqueos, había que esperar 24 horas para repetir la votación.

Más allá de estos avatares en consonancia con lo que ha ocurrido estos últimos años en la legislación audiovisual española, el CAC publica prestigiosos informes periódicos sobre diferentes aspectos comunicativos, vela por la pluralidad lingüística y cultural, tiene capacidad sancionadora, promueve normas de autorregulación, vela por el pluralismo de los medios y el cumplimiento de la misión de servicio público. 
Entre sus funciones, una muy importante que libera al gobierno autónomo de la responsabilidad de la concesión de las licencias de la TDT regional y local. El CAC emitió un dictamen preceptivo y vinculante, previo a la concesión de los 56 canales de TDT local privada por el gobierno de la Generalitat presidido por Pasqual Maragall, del PSC, que gobernaba en coalición con Esquerra Republicana de Catalunya, Iniciativa per Catalunya Verds-Esquerra Unida y Alternativa el 18 de julio de 2005. El poder de decisión del CAC y su independencia evitó que las concesiones estuvieran copadas por medios afines al ejecutivo autonómico, como ocurrió de forma generalizada en el resto de comunidades (López-Olano, 2015: 271-276).

El CAC tampoco se ha librado de las críticas, sobre todo después de la entrada pactada de los consejeros con perfil político Roger Loppacher -como presidente- y Daniel Sirera, que había sido presidente del Partit Popular de Catalunya (PPC). Ambos accedieron a la institución con los votos únicamente de CiU y PP. Y eso que como hemos visto, en la modificación de la Ley del CAC se mantuvo la prevención de que los miembros del Consell tuvieran experiencia profesional previa en el sector audiovisual, y que ofrecieran garantías plenas de independencia. 28

El acuerdo fue criticado por el Sindicat de Periodistes de Catalunya. ${ }^{29}$ La polémica ha ido más allá: después de la manifestación independentista del 11 de septiembre de 2012, el PPC pidió un informe al organismo regulador de la cobertura informativa realizada por TV3. Dos meses después, el informe solicitado determinó que "va haver respecte al pluralisme polític un cert equilibri pel que fa a la presència de les diferents opcions i opinions". ${ }^{30} \mathrm{El}$ PP calificó el informe de "sesgado", y acusó al organismo de ocultar los votos particulares de dos representantes del PSC y uno del PP, gracias al voto de calidad del presidente.

\section{Consejo Audiovisual de Andalucía (CAA) ${ }^{31}$}

También en Andalucía se creó un Consejo del Audiovisual autóctono, mediante la Ley 1/2004 de 17 de diciembre, de Creación del Consejo Audiovisual de Andalucía. ${ }^{32}$ Incluso se ha convertido en órgano de autogobierno en el nuevo Estatuto de Autonomía, ${ }^{33}$ tal como se contempla en su artículo 131:

1. El Consejo Audiovisual es la autoridad audiovisual independiente encargada de velar por el respeto de los derechos, libertades y valores constitucionales y estatutarios en los medios audiovisuales, tanto públicos como privados, en Andalucía, así como por el cumplimiento de la normativa vigente en materia audiovisual y de publicidad.

2. El Consejo Audiovisual velará especialmente por la protección de la juventud y la infancia en relación con el contenido de la programación de los medios de comunicación, tanto públicos como privados, de Andalucía.

3. Una ley del Parlamento regulará su composición, competencia y funcionamiento.

Está formado por 11 miembros, elegidos por el Parlamento de Andalucía por mayoría de tres quintos. El pleno del CCA es quien propone, de entre sus miembros, al presidente, que es 
nombrado por el Consejo de Gobierno. El mandato es de cinco años, renovable por otro período idéntico. En cuanto a sus funciones, son similares a las que disfruta su homólogo catalán, pero sin contar con su capacidad sancionadora. Vela por el pluralismo, la protección de los niños, también por una publicidad ajustada a derecho, garantiza el cumplimiento de servicio público, y emite informes de diversos temas. Entre ellos, también de las concesiones de licencias de radiodifusión y televisión. Por último, realiza, a instancias de las partes en conflicto, funciones arbitrales y de mediación. También existe una Oficina de defensa de la Audiencia. ${ }^{34}$

Aparte de estas dos instituciones, cabe repasar ahora otras comunidades autónomas españolas en las que también se reguló, o en algunos casos se puso incluso en marcha consejos reguladores o de autoridad audiovisual. En el caso de Madrid, se creó en abril de 2001 con funciones meramente consultivas. El mismo año se creó el Consejo Audiovisual de Navarra, órgano de referencia del Gobierno navarro con funciones de control, inspección y sanción. Con funciones meramente consultivas también se creó en Galicia el Consejo Asesor de las Telecomunicaciones y el Audiovisual a finales de 1999. Todas estas iniciativas fueron finalmente eliminadas excusándose en la crisis por los diferentes gobiernos del Partido Popular. Un último ejemplo: el Consell de l'Audiovisual de les Illes Balears fue creado, después de incluir el mandato en el artículo 77 del nuevo texto estatutario, por la Ley Balear 2/2010, de 7 de junio, durante la presidencia del socialista Francesc Antich, aunque no llegó a ponerse en marcha en la práctica.

Algo más tarde, aprovechando esta circunstancia, fue desprovista de competencias por la Ley General Audiovisual 5/2013 del 1 de octubre durante el gobierno popular de José Ramón Bauzá. El resultado es que el Consejo balear ha permanecido en un limbo legal, a pesar de la indicación estatutaria, mientras no ha cambiado la filiación política del gobierno autonómico.

\subsection{El marco legal en la Comunitat Valenciana}

El estado de las autonomías concibe éstas con amplias atribuciones en materia audiovisual, y la Comunitat Valenciana ha ido desarrollando su propia normativa, sin ser nunca la primera en hacerlo, un derecho que parece reservado a las comunidades históricas, especialmente a Catalunya y País Vasco, y con una tendencia al mimetismo en las leyes compartida con el resto de autonomías. Como norma marco actualmente en vigor, en la Comunidad Valenciana rige la Ley 1/2006 de 19 de abril, de la Generalitat, del Sector Audiovisual, 35 que ha sido calificada por el profesor Gavaldà como una humillación, "a la luz de lo realizado por el PPCV en RTVV a lo largo de once años" (2014a: 373).

En la Ley 1/2006 hay mandato de creación del Consell Audiovisual, aunque este también se incluiría en la reforma de l'Estatut. No hay referencias en cambio a lo que tiene que ver con la radio, el cine o la publicidad (Vidal Beltrán, 2011: 203-204). El hecho de incluir la creación de la Autoridad del Audiovisual en l'Estatut es destacable. Las dos normas, la Ley 1/2006 de la Generalitat del Sector Audiovisual, y la Ley Orgánica 1/2006 de 10 de abril fueron redactadas paralelamente en el tiempo, y esto pudo condicionar que la referencia al Consell Audiovisual entrara en ambos textos de esta manera. Finalmente, l'Estatut estableció en el artículo 56 que: 
Por ley de las Cortes Valencianas, aprobada por mayoría de tres quintas partes, se creará el Consell del Audiovisual de la Comunitat Valenciana, que velará por el respeto de los derechos, libertades y valores constitucionales y estatutarios en el ámbito de la comunicación y los medios audiovisuales en la Comunitat Valenciana. En cuanto a su composición, nombramiento, funciones y estatuto de sus miembros, igualmente habrá que ajustarse a lo que disponga la Ley.

Y en el preámbulo de la Ley del Audiovisual se especifica esto:

Por medio de la promulgación de una ley específica, se creará el Consell Audiovisual de la Comunitat Valenciana como órgano independiente que se regirá por la presente ley, por la propia ley por la que se cree, y por otras disposiciones aplicables. La ley de creación del Consell Audiovisual de la Comunitat Valenciana establecerá su composición, sus funciones y competencias que, junto con las que la presente ley señala, desarrollará para el mejor funcionamiento del sector audiovisual de la Comunitat Valenciana.

Y en el Capítulo II, artículo 5:

Mediante una ley específica se creará el Consejo del Audiovisual de la Comunitat Valenciana en el que se determinará su cometido, naturaleza y régimen jurídico, ámbito y principios de actuación, estructura orgánica y composición, estatuto de sus miembros, recursos económicos, organización y funcionamiento, personal a su servicio y relaciones con las instituciones de La Generalitat.

A pesar de estas referencias y del claro mandato estatutario, nueve años después de incluirse este punto, el anterior gobierno del Partido Popular de la Generalitat cumplió hasta el final con la tendencia a retrasar, minimizar o impedir la existencia de estos órganos reguladores, que se ha convertido en la política maestra del PP a nivel nacional y en el resto de comunidades respecto a la Alta Autoridad Audiovisual. También se ordenaba un Consorcio Audiovisual de la Comunitat, en el Capítulo III, y en el IV, un Registro General de Empresas Audiovisuales. Ninguno de estos órganos llegó a constituirse.

El Consell de l'Audiovisual hauria d'esdevenir una autèntica autoritat independent sobre tota classse d'operadors i concesionaris, de titularitat i/o gestió pública o privada, i amb competències reguladores i sancionadores sobre els continguts del sector, inclosos els formats i les vies de transmissió. El Consell, com és natural, hauria de vetlar pel compliment de la legislació. Però, des del nostre punt de vista, no es tracta només de fer cumplir una determinada normativa i de controlar els 
operadors que emeten al nostre territori, sinò d'atendre les prioritats derivades de l'interés públic i la responsabilitat davant la ciutadania. El Consell de l'Audiovisual Valencià hauria d'intervindre també en els procesos concessionals i supervisar les xarxes de telecomunicacions del nostre país. (Mollà i Orts, 2007: 470-471)

\section{Resultados. Previsiones de futuro}

Hasta el momento presente llega este recorrido por la situación de las autoridades de regulación que hay en España. Después del análisis comparativo, hemos podido comprobar en primer lugar el retraso en la creación en nuestro país de una herramienta considerada como fundamental para obtener unas televisiones públicas independientes y plurales, entre otras funciones. Tarde, mucho después que los países de nuestro entorno, y mal, ya que el macroregulador creado no cumple las expectativas. Hemos analizado sus actuaciones durante los dos años transcurridos desde su creación, algunas de calado en otros temas, pero que en ningún momento han abordado lo que claramente las leyes le exigen respecto al control del servicio público de RTVE.

El panorama no mejora en cuanto a la presencia en las autonomías de los órganos reguladores del audiovisual. Las dos únicas comunidades en las que está presente no se han librado de las críticas, pero en cualquier caso hay que remarcar que gran parte de las televisiones autonómicas y locales se quedan sin control en este mapa casi vacío. Y eso que numerosas y diferentes leyes, tanto a nivel estatal como autonómico, exigen la creación de los Consejos Audiovisuales.

La Comunidad Valenciana es un buen ejemplo: tanto el Estatut d'Autonomia como la ley audiovisual aún vigente, ambas del año 2006, se refieren a este organismo supervisor. La tendencia política del Partido Popular, marcada desde el gobierno de la nación y aplicada por sus barones territoriales, ha sido retrasar, suspender, minimizar y anular estos consejos en todas las ocasiones en las que ha sido posible. $\mathrm{Y}$ eso que estas leyes fueron promovidas y aprobadas, en algunos casos, por políticos del mismo partido.

Con el nuevo horizonte político en España surgido de las urnas a partir de las convocatorias electorales del año 2015, se puede esperar que vamos a ver cambios en muchos temas, también en el referido a las autoridades audiovisuales. Independientemente de los resultados aún inciertos a nivel nacional en el momento de acabar este artículo, recordemos que en el campo autonómico las comunidades de Aragón, Baleares, Castilla-La Mancha, Extremadura y Valencia han cambiado su signo político, desbancando las coaliciones de izquierda a los gobiernos del Partido Popular después de muchos años. Es previsible que con el recambio se ponga en marcha de nuevo la creación de los consejos, con lo que la situación puede alterarse en esta legislatura. Además en casi todas estas comunidades existen televisiones autonómicas en activo.

La excepción sería la Valenciana, donde como sabemos se cerró Canal 9 en 2013. Pero hay un consenso claro de los que participan en el Gobierno, PSOE y Compromís, con Podemos que lo 
apoya sin participar: todos coinciden en la necesidad de recuperar el servicio público, y en aprovechar la situación de tabula rasa para acometer una necesaria regeneración de la gobernanza en RTVV. En el documento de trabajo presentado por la Comisión de Expertos en Comunicación de las Universidades Valencianas ante las Cortes a propuesta del Foro Social del Audiovisual, entre otras muchas propuestas, también se valora como imprescindible la recuperación de la herramienta:

El Consell és un órgan imprescindible com a mecanisme per a vigilar la independència dels mitjans de comunicació públics. Només es pot garantir uns mitjans de comunicació públics independents si s’aconsegueix que els seus professionals treballen en llibertat, sense pressions de cap tipus. Per aquest motiu, es necessita un organisme regulador, que ordene el mercat audiovisual, amb autoritat i amb capacitat sancionadora, i que també supervise la concessió dels ajuts públics als mitjans valencians... (Comissió d’Experts en Comunicació de les Universitats Valencianes, 2015: 39)

La Ley 12/2015, de 29 de diciembre, para la recuperación del servicio público de radiodifusión y televisión de ámbito autonómico, ${ }^{36}$ pone plazos a la recuperación del servicio audiovisual, con el compromiso de que en el verano de 2016 entraría en vigor el nuevo estatuto de RTVV. En el borrador del proyecto de ley embrión de ese estatuto, recientemente presentado por Podemos y que en breve se debatirá en las Cortes, aparece incluso la propuesta de creación de un Consejo Ciudadano, que ejercería una función de control de la pluralidad de la futura televisión. ${ }^{37}$ En las tareas pendientes del legislador, queda también redactar una nueva ley del audiovisual, y poner en marcha, por fin, el Consell Valencià de l'Audiovisual.

En cuanto a la situación en el ámbito estatal español, si es una coalición de izquierdas la que accede finalmente al gobierno, es previsible que la puesta en marcha del CEMA contemplado en la LGCA vuelva a ponerse encima de la mesa. Recientemente, el colectivo Teledetodos presentó una ambiciosa propuesta de regeneración del Servicio Público de Radio, Televisión y Servicios interactivos. Entre sus recomendaciones aparece también la recuperación de un organismo regulador independiente, especializado y con atribuciones efectivas, en la cúspide del sistema de control:

El Consejo Audiovisual como gran regulador y vigilante del sistema audiovisual entero, en cada ámbito geográfico (estatal o autonómico); el Consejo Social como plataforma y vehículo de la participación de la sociedad civil en el servicio público a todas las escalas (estatal y regional), que debe jugar un papel esencial en la elección y control de los gestores; y finalmente el Consejo de Administración, que se configura como garante de la misión de servicio público, ejerciendo el control programático y presupuestario, y encomendando la gestión ejecutiva a la Dirección General, sin perjuicio del control último que corresponde naturalmente al Parlamento. (Teledetodos, 2015: 53) 
Este ambicioso informe se presentó a los diferentes partidos políticos de la oposición, ya antes de las Elecciones Generales del 20 de diciembre de 2015. Podemos, por ejemplo, anunció que lo adoptaría al $90 \%$ en caso de acceder al gobierno. ${ }^{38}$

\section{Conclusiones}

Ante el general consenso de los investigadores, expertos, profesionales y partidos de la izquierda, todo parece indicar que ha llegado el momento de reabrir el debate sobre la necesidad de recuperar unos consejos del audiovisual independientes, efectivos y con competencias reales. Hay que recordar que los enemigos de estos organismos de control han sido en primer lugar el Partido Popular, que sin explicitarlo en sus programas y aplicando en sus gobiernos el método del olvido y el retraso sin justificación, han provocado la minimización generalizada del recurso; y en segundo lugar, los operadores de televisión privados, principalmente el duopolio formado por Atresmedia y Mediaset España, a través de su lobby Unión de Televisiones Comerciales en Abierto (UTECA). Frente a las presiones del sector, se sitúa el clima de recuperación de la gobernanza adecuada para la televisión pública, y las bases de una regeneración que sitúe el sector audiovisual español por fin en consonancia con los países de su entorno. El recorrido de la aplicación en España de las recomendaciones europeas sobre organismos reguladores ha sido, como hemos podido comprobar, proceloso e irregular. Cabe estar vigilante a partir de ahora de su implantación en un futuro inmediato, lo que requerirá, sin duda, desarrollar ulteriores investigaciones al respecto.

\section{Bibliografía}

ARIÑO, M. (2011): "La regulación audiovisual en la era de la convergencia digital". Revista de los Estudios de Derecho y Ciencia Política de la UOC, n 7, páginas 1 a 7. Recuperado el 14 de abril de 2016 de: http://www.uoc.edu/idp/7/dt/esp/arino.pdf

BBC (2006). The BBC's Editorial Guidelines. Recuperado el 15 de diciembre de 2015 de: http://www.bbc.co.uk/editorialguidelines/guidelines/;

BELANDO GARÍN, B. (2014). Democracia y medios de comunicación. Cuestiones al hilo de la reforma de los medios de comunicación en el Reino Unido. En A. Boix Palop \& J.M. Vidal Beltrán (Eds.), La nueva regulación del audiovisual: medios,derechos y libertades, páginas 121 a 138). Navarra: Thomson Reuters Aranzadi.

BOIX PALOP, A. and Vidal Beltrán, J.M. (2014). La nueva regulación del audiovisual: medios, derechos y libertades. Navarra: Thomson Reuters Aranzadi.

BUSTAMANTE, E. (2014). El caso español: mutación ideológica de modelos. En M. Francés, J. Gavaldà Roca, G. Llorca Abad \& À. Peris Planes (Eds.), La televisión de la crisis ante el abismo digital, páginas 15 a 30. Barcelona: Gedisa. 
CABRAL, L y ROLDÁN, F. (2011): Competencia y regulación en el mercado audiovisual en España. Pamplona: IESE Business School. Recuperado el 15 de abril de 2016 de: http://bit.ly/1SbatBp

\section{COMISSIÓ D'EXPERTS EN COMUNICACIÓ DE LES UNIVERSITATS VALENCIANES}

(CECUV) (2015). Bases per a la renovació de l'espai comunicatiu valencià $i$ la restitució del servei públic de radiotelevisió. Recuperado el 31 de dicembre de 2015 de: http://bit.ly/1VplhBi

DE MORAGAS, M. and PRADO, E. (2000). La televisió pública a l'era digital. Barcelona: Pòrtic.

GARCÍA CASTILLEJO, A. (2013): “La regulación de los contenidos audiovisuales”. Revista TELOS, páginas 1 a 13. Recuperado el 12 de abril de 2016 de: https://goo.gl/f5gloU

GARCÍA LÓPEZ, J.L. (2014). La comisión nacional de los mercados y la competencia (CNMC) y sus funciones de supervisión sobre los contenidos audiovisuales. En A. Boix Palop \& J.M. Vidal Beltrán (Eds.), La nueva regulación del audiovisual: medios, derechos y libertades, páginas 153 a 172. Navarra: Thomson Reuters Aranzadi.

GAVALDÀ ROCA, J. (2014a). La calidad democrática y la regulación audiovisual. La radio y la televisión pública valenciana. En A. Boix Palop \& J.M. Vidal Beltrán (Eds.), La nueva regulación del audiovisual: medios, derechos y libertades, páginas 371 a 388. Navarra: Thomson Reuters Aranzadi.

GAVALDÀ ROCA, J. (2014). La política de la comunicación de la crisis. En M. Francés, J. Gavaldà Roca, G. Llorca Abad \& À. Peris Planes (Eds.), La televisión de la crisis ante el abismo digital, páginas 81 a 121. Barcelona: Gedisa.

GAVALDÀ ROCA, J. (2011). Contenidos de cantidad. En M. Francés (Ed.), Contenidos y formatos de calidad en la nueva televisión, páginas 69 a 98. Madrid: Instituto Radio Televisión Española/Publidisa.

LAKIDAIN, R. and PATTERSON, J.R. (2012). Consejo de informativos de TVE. Gestación y primeros años de historia. En M. Lamuedra Graván (Ed.), El futuro de la televisión pública. La necesaria alianza con la ciudadanía, páginas 101 a 128. Madrid: Popular.

LÓPEZ-OLANO, C. (2015). Los mecanismos de control de los informativos en las televisiones públicas. El caso de Canal 9. (Tesis de doctorado) Anne Arbor (Michigan): ProQuest. Recuperado el 10 de abril de 2016 de: http://bit.ly/1VpmZCO

LÓPEZ RICO, C. M. (2013): “RTVV y RTVE, diferentes modelos de autorregulación”, en Miguel Hernández Communication Journal, nº 4, páginas 21 a 34. Universidad Miguel Hernández, 
UMH (Elche-Alicante). Recuperado el 10 de abril de 2016 de: http://mhcj.es/2013/02/25/carmen lopez/

LOWE, G.F. and Steemers, J. (2012). Regaining the iniciative for public service media. Goteborg: Nordicom.

LINDE PANIAGUA, E. y VIDAL BELTRÁN, J.M. (2003). Derecho audiovisual. Madrid: Colex.

MARTÍNEZ OTERO, J.M. (2014) La CNMC y la protección de los menores en el audiovisual: asignaturas pendientes. En A. Boix Palop \& J.M. Vidal Beltrán (Eds.), La nueva regulación del audiovisual: medios, derechos y libertades, páginas 253 a 266. Navarra: Thomson Reuters Aranzadi.

MOLLÀ I ORTS, A. (2007). La televisió valenciana del segle XXI: nous factors $i$ noves funcions, autodiagnòstic $i$ anàlisi de prospectiva. València: Universitat de València.

TELEDETODOS (2015). Un nuevo modelo para un tiempo nuevo. España 2015: diagnóstico del servicio público de radio, televisión y servicios interactivos. Propuestas para una ciudadanía democrática. Recuperado el 31 de diciembre de 2015 de: http://teledetodos.es/index.php/dictamen-2015

TORNOS MAS, J. y DE CARRERAS I SERRA, L. (1999). Las autoridades de regulación de lo audiovisual, Madrid etc: Marcial Pons.

VIDAL BELTRÁN, J.M. (2011). "La reforma estatutaria y el régimen jurídico de los medios de comunicación audiovisuales en la comunidad valenciana". Revista Valenciana de Estudios Autonómicos, Vol. 56, páginas 162 a 229.

\section{Notas}

1 http://eur-lex.europa.eu/legalcontent/ES/TXT/PDF/?uri=CELEX:31989L0552\&qid=1425032908705\&from=EN [Consulta: 27/2/2015]

2 http://www.boe.es/buscar/act.php?id=BOE-A-2010-5292 [Consulta: 31/12/2015]

3 http://www.fcc.gov [Consulta:1/3/2015]

${ }^{4}$ http://www.csa.fr [Consulta:19/3/2015]

5 Pasaron de nueve a siete en la última reforma del año 2013, que también modificó la forma de nombrar a estos consejeros. El procedimiento aumenta la independencia del organismo regulador respecto a los poderes públicos, ya que requiere un amplio consenso sobre las personas electas. Una política radicalmente diferente de la que se hace en España en la misma época.

${ }^{6} \mathrm{http}: / /$ www.vertele.com/noticias/las-4-televisiones-publicas-mas-fuertes-de-europa-a-las-quepodria-imitar-tve/ [Consulta:15/6/2015] 
7 http://www.die-medienanstalten.de [Consulta:18/3/2015]

${ }^{8}$ http://www.agcom.it [Consulta:18/3/2015]

9 http://www.ofcom.org.uk [Consulta:19/3/2015]

${ }^{10}$ La sede de la BBC está situada en el icónico Broadcasting House de Londres, inaugurado en 1932, y sucesivamente ampliado a lo largo de la historia.

${ }^{11}$ https://www.boe.es/diario boe/txt.php?id=BOE-A-2013-5940 [Consulta: 1/9/15]

${ }^{12}$ http://www.cnmc.es [Consulta: 12/3/15]

${ }^{13}$ http://www.boe.es/buscar/act.php?id=BOE-A-2006-9958 [Consulta: 31/12/2015]

${ }^{14}$ http://www.boe.es/boe/dias/2008/06/30/pdfs/A28833-28843.pdf [Consulta: 19/3/15]

${ }^{15}$ http://www.cnmc.es/eses/Competencia/novedadesCompetencia/novedadesCompetenciadetal le.aspx?id=1408 [Consulta: $12 / 3 / 15]$

16 http://www.cnmc.es/es-

es/telecomunicacionesysaudiovisuales/novedadestelecomunicacionesysaudiovisuales/novedadest elecomunicacionesysaudiovisualesdetalle.aspx?id=3382 [Consulta: $12 / 3 / 15]$

17 http://www.cnmc.es/es-

es/telecomunicacionesysaudiovisuales/novedadestelecomunicacionesysaudiovisuales/novedadest elecomunicacionesysaudiovisualesdetalle.aspx?id=11438 [Consulta: 12/3/15]

18 http://www.cnmc.es/es-

es/telecomunicacionesysaudiovisuales/novedadestelecomunicacionesysaudiovisuales/novedadest elecomunicacionesysaudiovisualesdetalle. aspx?id=43718 [Consulta: 12/3/15]

${ }^{19} \mathrm{http} / /$ www.cnmc.es/Portals/0/Ficheros/Telecomunicaciones/Resoluciones/2014/Diciembre /141211_REQ-DTSA-1890-14-Mediaset\%20Salvame.pdf [Consulta: 12/3/15]

$20 \mathrm{http} / /$ tendencias.vozpopuli.com/television/4030-jorge-javier-vazquez-y-los-colaboradoresde-salvame-se-enfrentan-a-la-cnmc [Consulta: 12/3/15]

${ }^{21}$ http://www.cac.cat/web/actualitat/notespremsa/llistat.jsp?MTU\%3D\&MQ\%3D\%3D\&L3dIY i9hY3R1YWxpdGF0L25vdGVzcHJlbXNhL2xsaXN0YXRDb250ZW50 [Consulta: 22/5/15]

${ }^{22}$ La redacción de la norma crea cierta confusión, ya que TV3 por ejemplo también emite, además de en Catalunya, en la Comunidad Balear. La CNMC podría regular por tanto, sus emisiones.

${ }^{23}$ http://www.cac.cat [Consulta: 12/3/15]

${ }^{24}$ http://www.parlament.cat/activitat/cataleg/TL1.pdf [Consulta: 19/3/15]

${ }^{25}$ http://dogc.gencat.cat/ca/pdogc_canals_interns/pdogc_resultats_fitxa/?documentId=440333 \&language $=\mathrm{ca}$ ES\&action $=$ fitxa $[$ Consulta: $03 / 2 / 15$ ]

${ }^{26} \mathrm{https://www.cac.cat/pfw}$ files/cma/normativa_sa/Llei 2-2012.pdf [Consulta: 16/3/15]

27 http://fesp.org/index.php/noticias/item/3675-ciu-y-pp-pactan-la-reforma-del-cac [Consulta: $16 / 3 / 15]$

${ }^{28}$ La única experiencia conocida de Daniel Sirera en medios de comunicación o empresas audiovisuales, es haber sido articulista en "Libertad Digital".

${ }^{29} \underline{\mathrm{http}}$ ///www.eldiario.es/catalunya/11s 0 71393190.html [Consulta: 16/3/15]

${ }^{30}$ http://www.cac.cat [Consulta: 16/3/15] 
${ }^{31}$ http://www.consejoaudiovisualdeandalucia.es [Consulta: 03/2/15]

${ }^{32}$ http://www.consejoaudiovisualdeandalucia.es/sites/default/files/ley de creacion boe.pdf [Consulta: 28/2/2015]

${ }^{33}$ http://www.boe.es/buscar/act.php?id=BOE-A-2007-5825 [Consulta: 28/2/2015]

${ }^{34}$ http://www.consejoaudiovisualdeandalucia.es/oda/conoce-la-oda [Consulta: 28/2/2015]

${ }^{35}$ http://www.docv.gva.es/portal/ficha disposicion.jsp?id=26\&sig=2165/2006\&L=1\&url lista $=$ [Consulta: 12/2/2015]

36 http://www.docv.gva.es/datos/2015/12/31/pdf/2015 10477.pdf [Consulta: 31/12/2015]

${ }^{37}$ http:/ / www.eldiario.es/cv/Canal-priorizara-contratacion-antiguos-

trabajadores 0 504299895.html [Consulta: 16/04/2016]

38 http://www.vertele.com/noticias/como-sera-tve-tras-el-20-d-las-respuestas-de-los-partidos-alas-4-preguntas-clave/ [Consulta: 31/12/2015] 


\section{(c) (i) (2)}

Licencia Creative Commons

Miguel Hernández Communication Journal

mhcj.es

Forma de citar este artículo en las bibliografías

Carlos López Olano (2016): "Las autoridades de regulación audiovisual en España. Panorámica y perspectivas del control de la pluralidad", en Miguel Hernández Communication Journal, nº7, páginas 87 a 112. Universidad Miguel Hernández, UMH (Elche-Alicante). Recuperado el _ de de $20 \_$de: @ink del artículo en mhjournal.org] 